\title{
Internet of Things for smart living
}

\author{
Al-Sakib Khan Pathan ${ }^{1} \cdot$ Zubair Md. Fadlullah $^{2} \cdot$ Salimur Choudhury $^{3} \cdot$ Mohamed Guerroumi $^{4}$
}

Published online: 4 March 2019

(C) Springer Science+Business Media, LLC, part of Springer Nature 2019

Human beings are always in the pursuit of making their lives as smart (and easy) as possible. The term 'smart' means the involvement of some sort of intelligence in a system or entity. We cannot say that the people who came before us were not living smart. For instance, in the old ages, for distant communication, people used to use letters, pigeons, arrows, etc. That was then the most modern and smartest way of doing that. When the wired telephone came, that was the smartest way of communication in that era. When the wireless technologies came and later, Internet became a real impactful medium of doing the same types of tasks, we called that the smartest way of communication. Similarly, in many aspects of our life, we seek smartness, willingly or unwillingly, just to find some ease in doing the tasks that we must do. Though smartness has always been a part of human lifestyle, in today's era, in the scientific and technological field, the word has specific connotation. One of the most recent smart concepts is the Internet of Things (IoT), which is supposed to make many things in our life easy (or, a better term, 'easier'). While many types of theoretical research works are being carried out on different aspects of it, some researchers also amaze the people with their small-scale implementations of the

Al-Sakib Khan Pathan

spathan@ieee.org

Zubair Md. Fadlullah

zfadlullah@ieee.org

Salimur Choudhury

salimur.choudhury@lakeheadu.ca

Mohamed Guerroumi

guerroumi@gmail.com

1 Department of Computer Science and Engineering, Southeast University, Dhaka, Bangladesh

2 Graduate School of Information Sciences, Tohoku University, Sendai, Japan

3 Department of Computer Science, Lakehead University, Thunder Bay, Canada

4 Department of Computer Science, USTHB University, Algiers, Algeria futuristic ideas. There are indeed various barriers to materializing the vision to a reality as such type of interaction and communication among a wide variety of things or objects (as expected, mainly wirelessly) may also incur various kinds of security and privacy loopholes, Quality of Service (QoS) and Quality of Experience (QoE) issues, reliability issues, human and machine interaction issues, etc. The objective of this Special Issue (SI) is to capture and cover the significant latest advancements in all these areas circulating around or based on IoT.

A condition for the papers in this issue was that those must have had to be accepted and presented in the 2nd EAI International Conference on Smart Grid and Internet of Things (SGIoT 2018), held on July 11, 2018 at Niagara Falls, Canada. Hence, after the event, among the submitted papers (extended and revised), we could accept only three high quality papers. Each paper has been significantly extended and revised based on the comments received during the review process in the conference and also, in the second phase of journal reviews.

In the recent years, there have been many works on healthcare issues from various perspectives. In this SI, Onasanya and Elshakankiri contribute a paper entitled, "Smart Integrated IoT Healthcare System for Cancer Care" in which they focus on the IoT based healthcare system for cancer care services and business analytics/cloud services, and also propose the adoption and implementation of IoT/WSN (Internet of Things/Wireless Sensor Network) technology to augment the existing treatment options to deliver healthcare solution. They also propose a variety of frameworks and architectures to illustrate and support the functional IoT-based solution that is being utilized in their proposed smart healthcare solution for cancer care services. A positive point of this paper is that the authors also talk about the nature of cancer and its impact - they make differences between different types of tumors and what could turn into a cancer at a later stage. The comparative chart between the proposed system and other alternative systems is also a point of interest. This paper overall provides a good amount of information about 
the efforts of smartening the healthcare systems and services using IoT technologies.

The paper entitled, "Using IoT and Smart Monitoring Devices to Optimize the Efficiency of Large-Scale Distributed Solar Farms," by Shapsough et al. presents a novel architecture that utilizes Internet of Things (IoT) technologies for real-time monitoring of large-scale distributed solar farms. The architecture was derived based on a review of the latest trends and challenges in solar monitoring, as well as the latest breakthroughs in IoT technologies. This is an informative paper that also discusses the challenges faced by solar farms, Smart Solar Photovoltaic (PV) monitoring, the role of IoT for PV monitoring, and other associated issues. Besides presenting their architecture of the system, they present the results of their practical implementation scenario. The authors also mention that it would be possible to seamlessly integrate distributed solar farms into existing ecosystems such as Smart Grid by adopting a modular layer-based paradigm for the system architecture and using IoT technologies.

The third paper is entitled, "A Framework for Harmonizing Internet of Things (IoT) in Cloud: Analyses and Implementation," which is contributed by Islam et al. In this work, the authors have presented an idea to harmonize two sensors such that they could interact with each other and act on output produced by one of the sensors. The mechanism is such that one is to detect the existence of fire and the other one would act as the output of the fire detector sensor. This is used to control the door in case of existence of fire. In such a case, the sensed data are stored in the cloud as there is no local storage used. With experimental evaluations, the authors show that their implementation outperforms the existing alternative systems in this area.

As may be understood, all of these three contributions talk about smartening the environment of different types with the aid of IoT and associated technologies, which would be the driving force for the next wave of technical world. At least devices would cooperate in greater scale in an Internet-like infrastructure and that may not be simply called the extension of what we call the Internet today. Humans in the loop or involvement of the human controllers would be crucial in all such application scenarios as ultimately, most of the technological advancements are done to ease the life of human beings.

As the guest editors, we hope that even though we have been able to include only three contributions for this special issue, these would have significant impact in this area and researchers would find important information for their own works.

Best Wishes
The Guest Editors

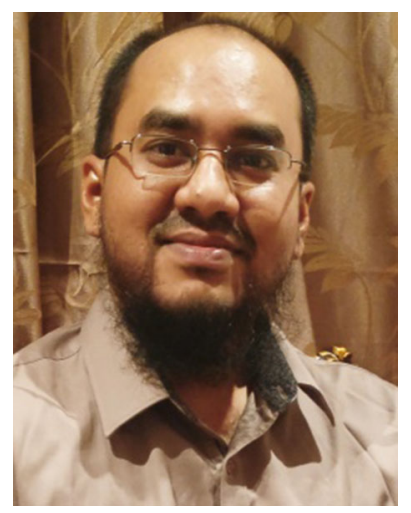

Al-Sakib Khan Pathan received Ph.D. degree in Computer Engineering in 2009 from Kyung Hee University, South Korea and B.Sc. degree in Computer Science and Information Technology from Islamic University of Technology (IUT), Bangladesh in 2003. He is currently an Associate Professor at the Computer Science and Engineering Department, Southeast University (SEU), Bangladesh. He is a Guest Lecturer for the STEP project at the Department of Technical and Vocational Education, Islamic University of Technology, Bangladesh since April 2018. His research interests include wireless sensor networks, network security, cloud computing, and e-services technologies. Currently he is also working on some multidisciplinary issues. He is a recipient of several awards/ best paper awards and has several notable publications in these areas. So far, he has delivered over 16 keynotes and invited speeches at various international conferences and events. He has served as a General Chair, Organizing Committee Member, and Technical Program Committee (TPC) member in numerous top-ranked international conferences/workshops like INFOCOM, GLOBECOM, ICC, LCN, GreenCom, AINA, WCNC, HPCS, ICA3PP, IWCMC, VTC, HPCC, SGIoT, etc. He was awarded the IEEE Outstanding Leadership Award for his role in IEEE GreenCom' 13 conference. He is currently serving as the Editor-in-Chief of International Journal of Computers and Applications, Taylor \& Francis, Associate Technical Editor of IEEE Communications Magazine, Editor of Ad Hoc and Sensor Wireless Networks, Old City Publishing, International Journal of Sensor Networks, Inderscience Publishers, The International Arab Journal of Information Technology (IAJIT), and Malaysian Journal of Computer Science, Associate Editor of International Journal of Computational Science and Engineering, Inderscience, Area Editor of International Journal of Communication Networks and Information Security, Guest Editor of many special issues of top-ranked journals, and Editor/ Author of 18 published books. He also serves as a referee of many prestigious journals. He received some awards for his reviewing activities like: one of the most active reviewers of IAJIT several times; Elsevier Outstanding Reviewer for Computer Networks, FGCS, and JNCA in multiple years. He is a senior member of IEEE.

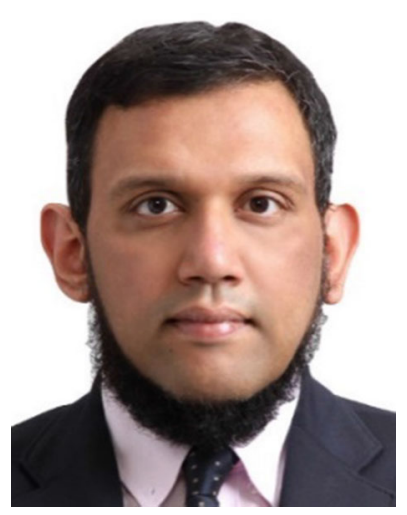

Zubair Md. Fadlullah received the B.Sc. (with Hons.) degree in Computer Science and Information Technology from the Islamic University of Technology (IUT), Bangladesh, in 2003, and the M.Sc. and Ph.D. degrees in Applied Information Science from Tohoku University, in 2008 and 2011, respectively. He is currently an associate professor in the Graduate School of Information Sciences, Tohoku University, Japan. His research interests include the areas of $5 \mathrm{G}$, smart grid, network security, intrusion detection, game theory, quality of security service provisioning mechanism, and deep learning. He received the Dean's Award and the President's Award from Tohoku University in 2011, the IEEE Asia Pacific Outstanding Researcher Award in 2015, and the NEC Foundation Prize for research contributions in 2016. He also received several best paper 
awards in the Globecom, IC-NIDC, and IWCMC conferences. He is a senior member of the IEEE.

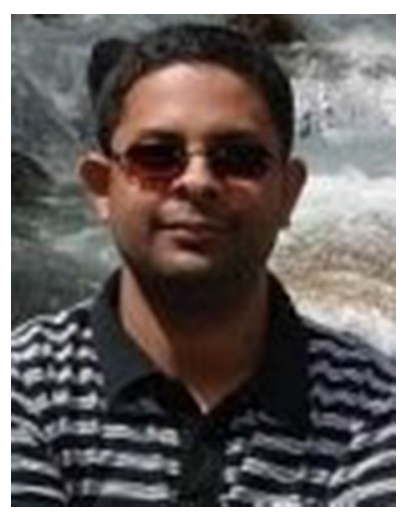

Salimur Choudhury is an Assistant Professor in the Department of Computer Science at Lakehead University, ON, Canada. $\mathrm{He}$ received Ph.D. in Computing from Queen's University, ON, Canada in 2012. His research interests include wireless networking and performance modeling and analysis of networking systems, optimization problems, wireless sensor network (WSN), and Internet of Things (IoT).

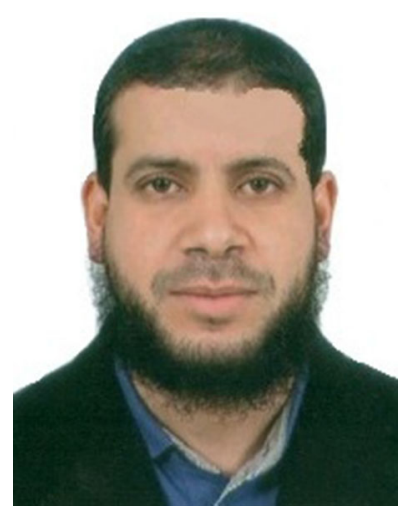

Mohamed Guerroumi is currently an Associate Professor at the Department of Computer Science, USTHB University, Algiers, Algeria. He received Ph.D. from USTHB University in 2014. He was a Data Network Senior Engineer at the Department of Core Network, Orascom Telecom Algeria from 2006 to 2013. From Jan 2014 to Oct 2016, he was a consultant as an IP Network and Security designer expert at DIVONA Company. As a Visiting Researcher, he visited the NDC Lab at IIUM, Kuala Lumpur, Malaysia, in 2014 and 2015, and the Network Research Group at The Centre for Distributed Computing, Networks and Security, Edinburgh Napier University in 2018. He is also serving as a Guest Editor of Annals of Telecommunications Journal and Chair for several workshops. He served as reviewer of numerous international journals like Elsevier Future Generation Computer Systems, IEEE Communications Magazine, Elsevier Computers \& Electrical Engineering, and Springer Wireless Personal Communications. He has served on the TPC of IEEE Globecom, IEEE ICC, ANT, ANT2, DependSys, ISCC, SpaCCS, and publication chair of SGIoT'18. His research interest is in performance evaluation of multihop wireless networks, focusing on developing performance evaluation frameworks and designing protocols in MAC and routing layers for wireless sensor networks and vehicular ad hoc network (VANET). Also, his current research interest lies in the security policies in wireless sensor networks, mobile, and software defined networks.

Publisher's Note Springer Nature remains neutral with regard to jurisdictional claims in published maps and institutional affiliations. 